\title{
Changing Profile of 7,519 Neurologic Outpatients Evaluated over 20 Years
}

\author{
Victor J. Del Brutto ${ }^{a}$ Daniel Tettamanti ${ }^{a}$ Oscar H. Del Brutto ${ }^{b}$ \\ aSchool of Medicine, Catholic University, and b Department of Neurological Sciences, Hospital-Clínica Kennedy, \\ Guayaquil, Ecuador
}

\section{Key Words}

Neurologic outpatients $\cdot$ Neurologic diseases $\cdot$ Ecuador •

Ambulatory patients $\cdot$ Neurologic disorders

\begin{abstract}
Introduction: There is scarce information on the demographic profile and diseases affecting neurologic outpatients. Methods: We evaluated 7,519 neurologic outpatients over 20 years at the outpatient clinic of the Department of Neurological Sciences, Hospital-Clínica Kennedy, Guayaquil, Ecuador. Results: Mean age was $48 \pm 19.4$ years, and $57.8 \%$ were women. Common reasons for consultation included headache (33.2\%), focal deficits (10.7\%), and seizures (9\%). The most common categories of neurologic diseases were cerebrovascular (10.6\%), degenerative (7.6\%), and traumatic (7.1\%). Diseases of uncertain/unknown etiology accounted for $50.2 \%$ of cases. Young patients were most often evaluated for headache and seizures, while elderly patients usually presented with focal deficits, movement disorders, or cognitive decline. We also found significant variations in the prevalence of neurologic diseases over the study years. While the prevalence of degenerative diseases increased from $5.7 \%$ in $1990-1994$ to $10.2 \%$ in $2005-2009$, that of infectious diseases steadily decreased from 3.7 to $2.1 \%$ over the study years. Conclusion: There was a dynamic pattern of neurologic diseases over the years. Nowadays, distribution
\end{abstract}

of neurologic symptoms and diseases in our population is more similar to that reported from the developed world than it was 20 years ago.

Copyright $\odot 2012$ S. Karger AG, Basel

\section{Introduction}

It is surprising how scarce information is on the profile of patients attending neurologic clinics, their most common disorders, and if there has been any change in the pattern of expression of neurologic diseases over the past years. Since more than $80 \%$ of the time spent by neurologists is office-based, evaluation of ambulatory patients is an important part of neurologic practice [1]. For proper medical assistance, we must be familiar with the most common disorders affecting this subset of neurologic patients, optimizing time, efforts and resources in their evaluation [2-4]. Most published series on neurologic outpatients come from Europe, where neurologists work within a socialized system of medical attention. Therefore, their findings may not be extrapolable to other regions and may not be even comparable to each other since a unified classification of neurologic disorders has not been used [5-15]. Moreover, there are less than a handful of studies focusing on neurologic outpatients from the developing world, where optimization of existing sani- 
tary resources - already stretched to the limits - are of particular importance [16-18]. Neurological disorders are top ranked among the most common causes of disability-adjusted life-years lost in adults living in developing countries [19]. Yet, much remains to be learned on the relative prevalence of most of these conditions, and the number of specialists needed to handle the burden of neurological diseases in these areas [20].

All published studies on neurologic outpatients are cross-sectional, providing no information on the variation of the prevalence of neurologic disorders over the years, or on the appearance of other neurologic disorders on the long-term follow-up [7, 12, 21, 22]. We present a large cohort of neurologic outpatients to analyze the changing pattern of disease expression of the different neurologic symptoms and neurologic disorders over two decades and to suggest a unified classification of these disorders for research purposes.

\section{Methods}

From January 1990 to December 2009, 7,519 persons aged 18 years or more were evaluated by a certified neurologist at the outpatient clinic of the Department of Neurological Sciences, HospitalClínica Kennedy, Guayaquil, Ecuador. Our institution is the largest center of private medicine in the city, serving people of middle and high socioeconomic status and, due to a number of agreements with public institutions, also serves individuals from the low economic income class, making our patients fairly representative of the population of the city. Patients were prospectively admitted into the cohort, using a unified format of clinical history that allowed comparisons by the end of the study. About $60 \%$ of our patients attended the clinic because they felt they had a neurologic problem, and $30 \%$ were referred from other physicians, including general practitioners, otolaryngologists, cardiologists, and neurosurgeons. The remaining $10 \%$ came to the outpatient clinic after been admitted and treated at the inpatient neurological service of our hospital. We analyzed data concerning the demographic profile of patients, reason for consultation, category of neurologic disease, diagnosis, presence of preexisting conditions, and neurologic disorders appearing on follow-up. Data was independently reviewed by two of the authors (V.J.D.B. and O.H.D.B.) and disagreements in data abstraction were resolved through discussion and consensus.

Patients were included into one of the following reasons for consultation: (a) headache and facial pain, (b) focal neurologic deficits of central origin, (c) seizures, (d) movement disorders, (e) dizziness, deafness and tinnitus, (f) weakness and/or sensory disturbances due to peripheral lesions, (g) cognitive decline, (h) pain in the neck, back and limbs, (i) loss of consciousness without focalizing signs, $(\mathrm{j})$ sleep disorders, and (k) non-neurologic (psychiatric and systemic clinical manifestations). For patients who had two or more symptoms, we chose the most relevant one, i.e. in patients with headache followed by loss of consciousness or a focal neurologic deficit, the latter were chosen as the main reasons for consultation.
The patients were then classified into one of the following major categories of neurologic disease: (a) cerebrovascular disorders, (b) degenerative diseases of the nervous system, (c) traumatic diseases, (d) diseases of structures anatomically related to the nervous system, (e) infections of the nervous system, (f) systemic, metabolic, toxic, medicamentous, or iatrogenic conditions, (g) neoplastic diseases of the central nervous system, (h) inflammatory or autoimmune disorders, (i) primary disturbances in CSF circulation, (j) congenital malformations or diseases, and (k) diseases of uncertain/unknown etiology. Finally, a specific diagnosis was done whenever possible.

After the initial subsequent visit programmed to review requested examinations and neurodiagnostic tests, a total of 2,864 patients were scheduled for further follow-up. We did not schedule patients who were referred to other specialists, as well as most patients with primary or post-traumatic headache, benign positional vertigo, vasovagal syncope, muscle contractures, sleep disorders, and those with non-neurologic complains.

We correlated the demographic profile of patients included in each of the main reasons for consultation and in each of the major categories of neurologic diseases to evaluate the presence of changes in any of these variables or in the prevalence of neurologic diseases over the years. Ethical aspects of the investigation as well as confidentiality of the names of the patients included in this cohort were assured by the IRB of our institution. $\chi^{2}$ and Fisher's exact tests were used to assess the significance of the difference between the diverse variables analyzed. Differences were considered significant if $\mathrm{p}<0.05$. Statistical analysis of data was carried out using EpiInfo-2000 software (Centers for Disease Control, Atlanta, Ga., USA).

\section{Results}

Table 1 summaries the demographic profile of patients as well as reasons for consultation and the category of neurologic disease in which they were included. In brief, mean age was $48 \pm 19.4$ years, and $57.8 \%$ were women. Two-thirds of the patients were younger than 60 years of age and less than $7 \%$ were older than 80 years. Classification of patients into four 5 -year periods according to the year of first evaluation showed a homogenous influx of patients over the study years.

Besides metabolic work-ups, X-ray films, ultrasound studies and immune diagnostic tests, 5,309 patients underwent one or more neurodiagnostic tests, including neuroimaging studies in 5,036 (67\%), EEGs in 1,062 (14.1\%), neurophysiological studies in 194 (2.6\%), and spinal taps in 190 (2.5\%). Some patients underwent biopsy of brain lesions, peripheral nerves or muscles. A total of 1,913 patients (67\% of 2,864 scheduled for follow-up) returned to the clinic and were followed up for a mean of $37.9 \pm 45.5$ months (range 2-240).

The percentage of patients within the diverse categories of neurologic diseases according to the reason for 
consultation is detailed in table 2, demographic profiles of patients according to the reason for consultation and the major category of neurologic disease are summarized in tables 3 and 4, and specific diagnoses (in 6,764 patients with neurologic disorders) are listed in table 5.

\section{Reasons for Consultation}

These included headache or facial pain in 2,496 patients, focal neurologic deficits of central origin in 804 , seizures in 680 , movement disorders in 579 , dizziness, deafness or tinnitus in 559, weakness and/or sensory abnormalities due to peripheral lesions in 505, cognitive decline in 379, pain in the neck, back or limbs in 356, loss of consciousness without focalizing signs in 326 , and sleep disorders in 80 . The remaining 755 patients had non-neurologic complains.

There were significant differences in the prevalence of some of the reasons for consultation according to the age of patients and to the year in which they were admitted into the cohort. In particular, we noted significant increases in the prevalence of focal neurologic deficits $(\mathrm{p}<$ 0.001 ), movement disorders ( $\mathrm{p}<0.001)$, and cognitive decline $(\mathrm{p}<0.001)$ with advanced age. In contrast, the prevalence of patients consulting for headache $(\mathrm{p}<0.001)$ and seizures $(\mathrm{p}<0.001)$ decreased with age (fig. 1$)$. The number of patients with movement disorders $(p<0.02)$ and cognitive decline $(\mathrm{p}<0.001)$ significantly increased, while the prevalence of patients presenting with seizures $(\mathrm{p}<0.01)$ decreased over the study years (fig. 2$)$.

\section{Categories of Neurologic Diseases}

Of the 6,764 patients with neurologic diseases, 3,588 had diseases of uncertain/unknown etiology, 800 had cerebrovascular disorders, 570 had degenerative diseases, 535 had traumatic diseases, 458 had diseases of structures anatomically related to the nervous system, 295 had infections of the nervous system, 194 had systemic, metabolic, toxic, medicamentous, or iatrogenic conditions, 162 had neoplastic diseases, 80 had inflammatory or autoimmune disorders, 46 had primary disturbances in CSF circulation, and 36 had congenital malformations or diseases.

Some of the major categories of neurologic disorders increased in prevalence with advancing age, in particular cerebrovascular disorders $(p<0.001)$ and degenerative diseases $(\mathrm{p}<0.001)$. Others, such as diseases of unknown etiology $(p<0.001)$, traumatic diseases $(p<0.01)$, and infections of the nervous system $(p<0.001)$ reduced in prevalence with age (fig. 1). The prevalence of degenerative diseases of the nervous system markedly increased over the study years $(\mathrm{p}<0.001)$, while the prevalence of
Table 1. Characteristics of 7,519 neurologic outpatients evaluated over 20 years (values in parentheses are percentages)

\begin{tabular}{|c|c|}
\hline Mean age $\pm S D$, years & $48 \pm 19.4$ \\
\hline Females & $4,346(57.8)$ \\
\hline \multicolumn{2}{|l|}{ Age group, years } \\
\hline $18-39$ & $3,063(40.7)$ \\
\hline $40-59$ & $2,103(28)$ \\
\hline $60-79$ & $1,875(24.9)$ \\
\hline $80-99$ & $478(6.4)$ \\
\hline \multicolumn{2}{|l|}{ Year of evaluation } \\
\hline 1990-1994 & $1,918(25.5)$ \\
\hline 1995-1999 & $1,841(24.5)$ \\
\hline $2000-2004$ & $1,935(25.7)$ \\
\hline $2005-2009$ & $1,825(24.3)$ \\
\hline \multicolumn{2}{|l|}{ Reason for consultation } \\
\hline Headache and facial pain & $2,496(33.2)$ \\
\hline Focal neurologic deficits & $804(10.7)$ \\
\hline Seizures & $680(9)$ \\
\hline Movement disorders & $579(7.7)$ \\
\hline Dizziness, deafness, tinnitus & $559(7.4)$ \\
\hline Peripheral weakness/sensory & \\
\hline disturbances & $505(6.4)$ \\
\hline Cognitive decline & $379(5)$ \\
\hline Pain in the neck, back or limbs & $356(4.7)$ \\
\hline Loss of consciousness & $326(4.3)$ \\
\hline Sleep disorders & $80(1.1)$ \\
\hline Non-neurologic & $755(10)$ \\
\hline \multicolumn{2}{|l|}{ Category of neurologic disease } \\
\hline Unknown etiology & $3,588(47.7)$ \\
\hline Cerebrovascular disorders & $800(10.6)$ \\
\hline Degenerative diseases & $570(7.6)$ \\
\hline Traumatic diseases & $535(7.1)$ \\
\hline Diseases of structures anatomically & \\
\hline related to the nervous system & $458(6.1)$ \\
\hline Infections of the nervous system & $295(3.9)$ \\
\hline Systemic, toxic, metabolic disorders & $194(2.6)$ \\
\hline Neoplastic diseases of CNS & $162(2.2)$ \\
\hline Inflammatory/autoimmune disorders & $80(1.1)$ \\
\hline Disturbances in CSF circulation & $46(0.6)$ \\
\hline Congenital malformations or diseases & $36(0.5)$ \\
\hline Non-neurologic & $755(10)$ \\
\hline
\end{tabular}

traumatic diseases $(\mathrm{p}<0.001)$ and infections of the nervous system $(\mathrm{p}<0.001)$ decreased over the years of the study (fig. 2).

\section{Specific Diagnoses}

The top 15 specific diagnoses in our patients were: migraine/tension-type headache in 1,728 (23\%), cerebral infarction in 516 (6.9\%), benign positional vertigo in 385 (5.1\%), cryptogenic epilepsy in 334 (4.4\%), post-traumatic headache in 271 (3.6\%), Alzheimer's disease/mild cognitive impairment in 205 (2.7\%), Parkinson's disease in 198 
Table 2. Percentage of patients included in the different categories of neurologic diseases according to the reason for consultation

\begin{tabular}{|c|c|c|c|c|c|c|c|c|c|c|c|c|}
\hline Reason for consultation & Syndromatic/anatomic diagnosis & $\begin{array}{l}\text { Un- } \\
\text { known }\end{array}$ & $\begin{array}{l}\text { Vas- } \\
\text { cular }\end{array}$ & $\begin{array}{l}\text { Dege- } \\
\text { nera- } \\
\text { tive }\end{array}$ & $\begin{array}{l}\text { Trau- } \\
\text { matic }\end{array}$ & $\begin{array}{l}\text { Struc- } \\
\text { ture- } \\
\text { related }\end{array}$ & $\begin{array}{l}\text { Infec- } \\
\text { tious }\end{array}$ & $\begin{array}{l}\text { Systemic/ } \\
\text { metabolic }\end{array}$ & $\begin{array}{l}\text { Neo- } \\
\text { plastic }\end{array}$ & $\begin{array}{l}\text { Inflamma- } \\
\text { tory auto- } \\
\text { immune }\end{array}$ & $\begin{array}{l}\text { Abnor- } \\
\text { mal CSF } \\
\text { flow }\end{array}$ & $\begin{array}{l}\text { Con- } \\
\text { geni- } \\
\text { tal }\end{array}$ \\
\hline \multirow{3}{*}{$\begin{array}{l}\text { Headache and facial } \\
\text { pain }(n=2,496)\end{array}$} & primary headache $(\mathrm{n}=1,820)$ & 100 & - & - & - & - & - & - & - & - & - & - \\
\hline & secondary headache $(n=534)$ & - & 5.6 & - & 55.4 & 10.1 & 5.6 & 9.2 & 9.6 & - & 3.6 & 0.9 \\
\hline & facial pain $(n=142)$ & 67.6 & - & - & 4.2 & 12.7 & 4.9 & 4.9 & 1.4 & 4.3 & - & - \\
\hline \multirow{2}{*}{$\begin{array}{l}\text { Focal neurologic } \\
\text { deficits }(\mathrm{n}=804)\end{array}$} & hemispheric/brainstem $(\mathrm{n}=702)$ & - & 84.3 & - & 3.3 & - & 1.3 & 0.1 & 4.7 & 6 & - & 0.3 \\
\hline & spinal cord $(n=102)$ & 7.8 & 2.9 & 43.1 & 14.7 & 6.9 & 8.8 & 1 & 5.9 & 5.9 & - & 3 \\
\hline \multirow[t]{2}{*}{ Seizures $(n=680)$} & primary/cryptogenic $(\mathrm{n}=368)$ & 100 & - & - & - & - & - & - & - & - & - & - \\
\hline & secondary $(n=312)$ & - & 18.9 & - & 1.3 & - & 57.1 & 4.8 & 11.2 & 0.3 & - & 6.4 \\
\hline \multirow{4}{*}{$\begin{array}{l}\text { Movement disorders } \\
(\mathrm{n}=579)\end{array}$} & tremor/rigidity $(\mathrm{n}=318)$ & 30.8 & 1.3 & 64.5 & - & - & 0.9 & 2.2 & 0.3 & - & - & - \\
\hline & dystonia/spams/myoclonus/tics $(\mathrm{n}=129)$ & 50.4 & 2.3 & 36.4 & - & - & 0.8 & 8.5 & - & - & - & 1.6 \\
\hline & incoordination/abnormal gait $(\mathrm{n}=125)$ & 35.2 & 36.8 & 3.2 & - & - & 1.6 & 4.8 & 6.4 & 1.6 & 10.4 & - \\
\hline & chorea/athetosis/ballismus $(\mathrm{n}=7)$ & - & 42.9 & 57.1 & - & - & - & - & - & - & - & - \\
\hline \multirow{2}{*}{$\begin{array}{l}\text { Dizziness, deafness, } \\
\text { and tinnitus }(\mathrm{n}=559)\end{array}$} & labyrinthine disorders $(n=531)$ & 80.2 & - & - & 5.5 & 14.3 & - & - & - & - & - & - \\
\hline & VIII cranial nerve lesions $(\mathrm{n}=28)$ & 32.1 & 32.1 & - & - & - & - & - & 32.1 & - & - & 3.7 \\
\hline \multirow{3}{*}{$\begin{array}{l}\text { Weakness or sensory } \\
\text { disturbances } \\
(n=505)\end{array}$} & cranial nerve lesions $(n=244)$ & 88.1 & - & - & 1.2 & 3.3 & 2 & 5 & 0.4 & - & - & - \\
\hline & pheripheral nerve lesions $(\mathrm{n}=204)$ & 18.1 & - & 3.9 & 8.8 & 43.7 & 5.9 & 18.1 & - & 1.5 & - & - \\
\hline & neuromuscular lesions $(\mathrm{n}=57)$ & 61.4 & - & 12.3 & - & 7 & 3.5 & 10.5 & - & 5.3 & - & - \\
\hline \multirow{4}{*}{$\begin{array}{l}\text { Cognitive decline } \\
(\mathrm{n}=379)\end{array}$} & dementia/cognitive impairment $(n=308)$ & - & 7.2 & 81.5 & 1.6 & - & 0.3 & 0.3 & 4.2 & 0.3 & 4.6 & - \\
\hline & acute amnesia $(\mathrm{n}=53)$ & 98.1 & 1.9 & - & - & - & - & - & - & - & - & - \\
\hline & acute confusional state $(\mathrm{n}=15)$ & 26.6 & 6.7 & - & 6.7 & - & - & 60 & - & - & - & - \\
\hline & mental retardation $(\mathrm{n}=3)$ & - & - & - & - & - & - & - & - & - & - & 100 \\
\hline $\begin{array}{l}\text { Pain in the neck, } \\
\text { back, and limbs } \\
(\mathrm{n}=356)\end{array}$ & pain in the neck/back/limbs $(\mathrm{n}=356)$ & 27.5 & - & - & 3.7 & 56.8 & 7 & 0.8 & - & 4.2 & - & - \\
\hline \multirow{3}{*}{$\begin{array}{l}\text { Loss of consciousness } \\
(\mathrm{n}=326)\end{array}$} & structural coma $(\mathrm{n}=172)$ & - & 15.7 & - & 70.9 & - & 6.4 & 4.7 & 1.7 & 0.6 & - & - \\
\hline & metabolic coma $(\mathrm{n}=7)$ & - & - & - & - & - & - & 100 & - & - & - & - \\
\hline & syncope $(\mathrm{n}=147)$ & 90.5 & - & - & - & - & - & 9.5 & - & - & - & - \\
\hline $\begin{array}{l}\text { Sleep disorders } \\
(\mathrm{n}=80)\end{array}$ & sleep disorders $(n=80)$ & 100 & - & - & - & - & - & - & - & - & - & - \\
\hline \multirow{2}{*}{$\begin{array}{l}\text { Non-neurologic } \\
(\mathrm{n}=755)\end{array}$} & psychiatric manifestations $(\mathrm{n}=186)$ & 100 & - & - & - & - & - & - & - & - & - & - \\
\hline & non-specific $(n=569)$ & 100 & - & - & - & - & - & - & - & - & - & - \\
\hline
\end{tabular}

(2.6\%), symptomatic neurocysticercosis in $190(2.5 \%)$, Bell's palsy in 166 (2.2\%), protrusion of intervertebral discs in $152(2 \%)$, vasovagal syncope in $133(1.8 \%)$, intracranial hemorrhage in $126(1.7 \%)$, muscle contracture in $94(1.3 \%)$, essential tremor in $86(1.1 \%)$, and cerebral concussion in 86 (1.1\%). These 15 diseases represented $69 \%$ of 7,764 patients with neurologic disorders included in the cohort.

\section{Preexisting Conditions}

A total of 270 patients (mean age $58.2 \pm 18.8$ years; $46.7 \%$ women) have had symptomatic unrelated neurologic diseases that occurred from months to years before the initial visit. Most common preexisting conditions were: symptomatic cerebral infarction in 54 , pro- trusion of intervertebral discs in 27 , benign positional vertigo in 25 , Bell's palsy in 23 , peripheral neuropathy in 21 , migraine-tension type headache in 17 , and cognitive impairment in 13. Of the 54 patients with an old symptomatic cerebral infarction, 16 consulted for a new ischemic stroke, 7 for cognitive impairment, and 6 for Parkinson's disease. Six out of 13 patients having a preexisting cognitive impairment consulted for Parkinson's disease, and 3 of 5 patients with preexisting Parkinson's disease consulted for cognitive impairment. Interestingly, most patients with preexisting primary headache consulted for benign positional vertigo, and most of the patients with preexisting benign positional vertigo consulted for primary headache. 

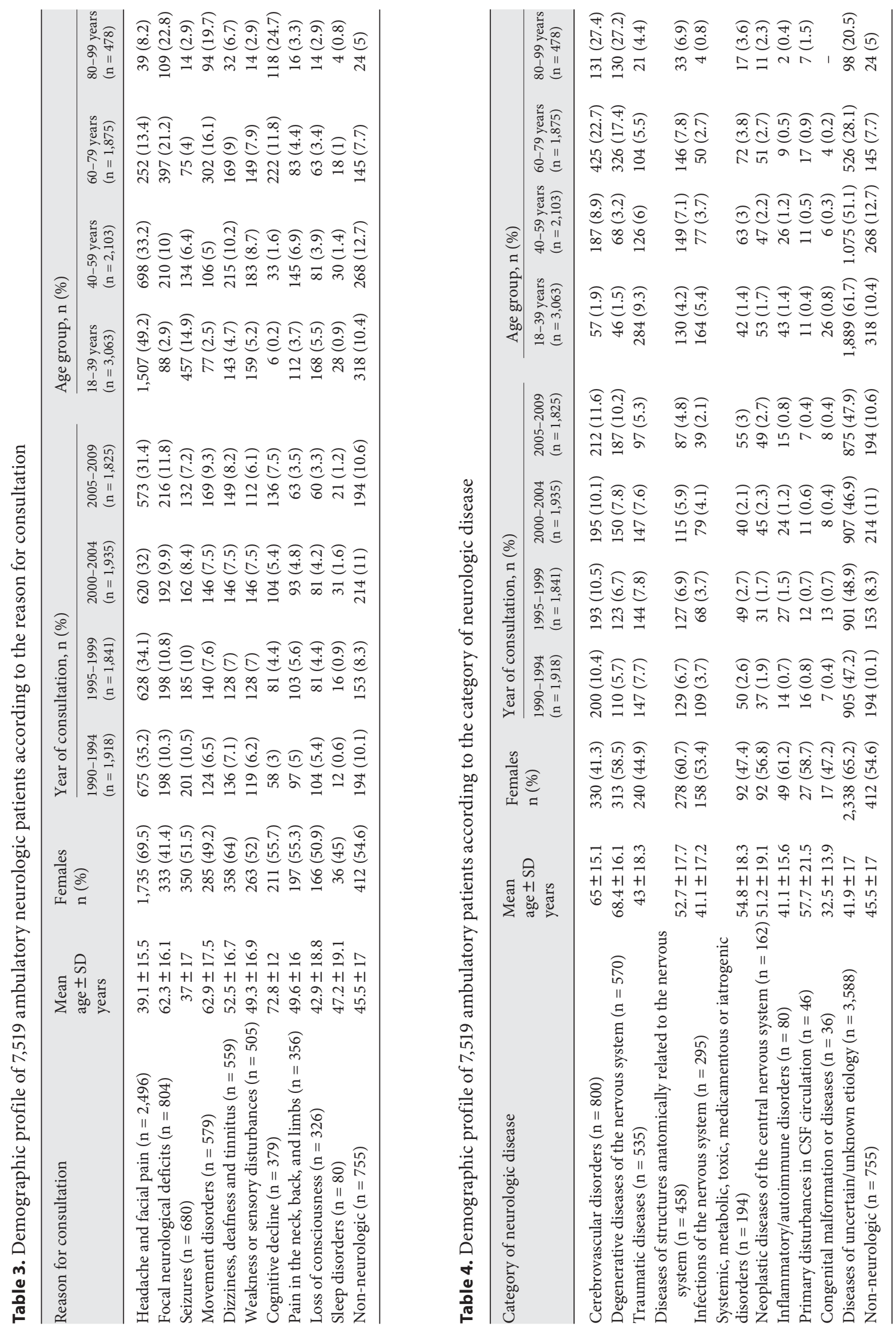
Table 5. Specific diagnosis in 6,674 ambulatory patients with neurologic diseases

\begin{tabular}{|c|c|c|}
\hline $\begin{array}{l}\text { Category of neurologic } \\
\text { disease }\end{array}$ & General diagnosis & Specific diagnosis \\
\hline \multirow[t]{3}{*}{$\begin{array}{l}\text { Cerebrovascular } \\
\text { disorders }(n=800)\end{array}$} & ischemic stroke (516) & $\begin{array}{l}\text { cerebral infarction due to: hypertensive arteriolopathy (215), large-vessel disease (42), } \\
\text { cardiogenic brain embolism (40), other causes (14), unknown cause (205) }\end{array}$ \\
\hline & hemorrhagic stroke (126) & $\begin{array}{l}\text { intracranial hemorrhage due to: hypertensive arteriolopathy (48), rupture of aneurysm/vascular } \\
\text { malformation (38), other causes (11), unknown cause (29) }\end{array}$ \\
\hline & $\begin{array}{l}\text { other cerebrovascular diseases } \\
(158)\end{array}$ & $\begin{array}{l}\text { transient ischemic attacks (59), undetermined stroke (31), unruptured aneurysm/vascular } \\
\text { malformation (46), ischemic optic neuropathy (17), venous thromboses (5) }\end{array}$ \\
\hline \multirow{3}{*}{$\begin{array}{l}\text { Degenerative diseases } \\
\text { of the nervous system } \\
(\mathrm{n}=570)\end{array}$} & $\begin{array}{l}\text { degenerative diseases of basal } \\
\text { ganglia (254) }\end{array}$ & $\begin{array}{l}\text { Parkinson's disease (198), blepharospasm/Meige's syndrome (15), Tourette's syndrome (10), } \\
\text { Writter's cramps (7), other/unclassified degenerative diseases of basal ganglia (24) }\end{array}$ \\
\hline & $\begin{array}{l}\text { degenerative diseases of cerebral } \\
\text { cortex (251) }\end{array}$ & $\begin{array}{l}\text { Alzheimer's disease (110), mild cognitive impairment (95), mixed dementia (38), Pick's } \\
\text { disease (7), Lewy's body disease (1) }\end{array}$ \\
\hline & other degenerative diseases (65) & $\begin{array}{l}\text { motor neuron disease (39), peroneal muscular atrophy (10), muscular dystrophies (6), other/ } \\
\text { unclassified degenerative diseases (10) }\end{array}$ \\
\hline \multirow[t]{2}{*}{$\begin{array}{l}\text { Traumatic diseases } \\
(\mathrm{n}=535)\end{array}$} & head trauma (489) & $\begin{array}{l}\text { post-traumatic headache (271), cerebral concussion (86), hemorrhagic contusion (54), } \\
\text { extracerebral hematomas (39), post-traumatic vertigo (29), other/unclassified manifestations of } \\
\text { head trauma (10) }\end{array}$ \\
\hline & $\begin{array}{l}\text { trauma of the spinal cord or } \\
\text { peripheral nerves }(46)\end{array}$ & $\begin{array}{l}\text { contusion of the spinal cord (15), post-traumatic neuropathy (15), whiplash syndrome (11), } \\
\text { other (4) }\end{array}$ \\
\hline \multirow{3}{*}{$\begin{array}{l}\text { Diseases of structures } \\
\text { anatomically related to } \\
\text { the nervous system } \\
(\mathrm{n}=458)\end{array}$} & diseases of vertebra (235) & $\begin{array}{l}\text { protrusion of intervertebral disc (152), osteoarthrotic myelopathy (45), lumbar stenosis (11), } \\
\text { vertebral listhesis (10), other/unclassified diseases of vertebra (17) }\end{array}$ \\
\hline & $\begin{array}{l}\text { diseases of craniofacial structures } \\
(156)\end{array}$ & $\begin{array}{l}\text { otosclerosis (71), sinusitis (48), Costen syndrome (14), mastoiditis (5), other/unclassified } \\
\text { diseases of craniofacial structures (18) }\end{array}$ \\
\hline & diseases of soft tissues (67) & entrapment neuropathies (60), other/unclassified diseases of soft tissues (7) \\
\hline \multirow{4}{*}{$\begin{array}{l}\text { Infections of the nervous } \\
\text { system }(n=295)\end{array}$} & parasitic infections (196) & neurocysticercosis (190), toxoplasmosis (6) \\
\hline & viral infections (31) & $\begin{array}{l}\text { viral encephalitis (9), viral meningitis (6), transverse myelitis (5), other/unclassified viral } \\
\text { infections (11) }\end{array}$ \\
\hline & bacterial infections (19) & pyogenic meningitis (9), neurotuberculosis (8), other/unclassified bacterial infections (2) \\
\hline & $\begin{array}{l}\text { other infections/sequelae of } \\
\text { remote infection ( } 49 \text { ) }\end{array}$ & post-herpetic neuralgia (32), Guillain-Barré syndrome (11), other/unclassified infections (6) \\
\hline \multirow{2}{*}{$\begin{array}{l}\text { Systemic, metabolic, } \\
\text { toxic, medicamentous, } \\
\text { or iatrogenic conditions } \\
(\mathrm{n}=194)\end{array}$} & $\begin{array}{l}\text { systemic/metabolic conditions } \\
(132)\end{array}$ & $\begin{array}{l}\text { headache related to arterial hypertension (42), diabetic neuropathy (35), cardiogenic } \\
\text { syncope (12), hypoxic-ischemic encephalopathy (10), hypokalemic periodic paralysis (5), } \\
\text { hypoglycemic coma (5), paraneoplastic syndromes (5), other systemic/metabolic conditions (18) }\end{array}$ \\
\hline & $\begin{array}{l}\text { toxic/medicamentous/iatrogenic } \\
\text { disorders }(62)\end{array}$ & $\begin{array}{l}\text { drug-induced movement disorders (15), seizures due to substance abuse (10), trigeminal } \\
\text { neuropathy due to dental manipulation (8), drug-induced acute confusional states }(7) \text {, } \\
\text { intracranial hypotension after spinal tap (4), other toxic/medicamentous/iatrogenic } \\
\text { disorders ( } 22)\end{array}$ \\
\hline \multirow{2}{*}{$\begin{array}{l}\text { Neoplastic diseases of } \\
\text { the central nervous } \\
\text { system }(n=162)\end{array}$} & primary brain tumor (144) & $\begin{array}{l}\text { gliomas (40), pituitary adenomas (32), meningiomas (31), neurinomas (9), other primary brain } \\
\text { tumors (20), uncharacterized primary brain tumor (12) }\end{array}$ \\
\hline & metastatic diseases (18) & intracranial metastases (17), meningeal sarcomatoses (1) \\
\hline \multirow{2}{*}{$\begin{array}{l}\text { Inflammatory and } \\
\text { autoimmune disorders } \\
(\mathrm{n}=80)\end{array}$} & demyelinating diseases (54) & $\begin{array}{l}\text { multiple sclerosis (19), optic neuritis (18), transverse myelitis (5), other/unclassified } \\
\text { demyelinating diseases (12) }\end{array}$ \\
\hline & other inflammatory diseases (26) & $\begin{array}{l}\text { chronic fatigue syndrome (15), giant-cell arteritis (6), polymyositis (3), granulomatous diseases } \\
\text { (2) }\end{array}$ \\
\hline Primary disturbances in & abnormal reabsorption of CSF (34) & normal pressure hydrocephalus (26), pseudotumor cerebri (8) \\
\hline CSF circulation $(n=46)$ & abnormal circulation of CSF (12) & primary hypertensive hydrocephalus (12) \\
\hline \multirow{2}{*}{$\begin{array}{l}\text { Congenital } \\
\text { malformation or disease } \\
(36)\end{array}$} & $\begin{array}{l}\text { structural malformation of CNS } \\
(24)\end{array}$ & $\begin{array}{l}\text { congenital leptomeningeal cysts (14), schizencephaly (3), other structural malformations of } \\
\text { CNS (7) }\end{array}$ \\
\hline & $\begin{array}{l}\text { other congenital malformation or } \\
\text { disease (12) }\end{array}$ & perinatal ischemic encephalopathy (5), facomatoses (3), other congenital malformations (4) \\
\hline $\begin{array}{l}\text { Diseases of uncertain/ } \\
\text { unknown etiology } \\
(3,558)\end{array}$ & & $\begin{array}{l}\text { migraine/tension-type headache (1,728), benign positional vertigo (385), cryptogenic epilepsy } \\
\text { (334), Bell's palsy (166), vasovagal syncope (133), muscle contractures (94), other primary } \\
\text { headaches ( } 92) \text {, essential tremor (86), insomnia/hypersomnia/parasomnia (80), idiopathic facial } \\
\text { pain (54), transient global amnesia (52), senile gait (44), trigeminal neuralgia (42), idiopathic } \\
\text { peripheral neuropathy (39), idiopathic peripheral neuropathy (36), primary epileptic syndromes } \\
\text { (34), myokymias (34), idiopathic ophthalmoplegia (34), Ménière's disease (32), hemifacial spasm } \\
\text { (27), myasthenia gravis (26), other diseases of uncertain/unknown etiology (56) }\end{array}$ \\
\hline
\end{tabular}




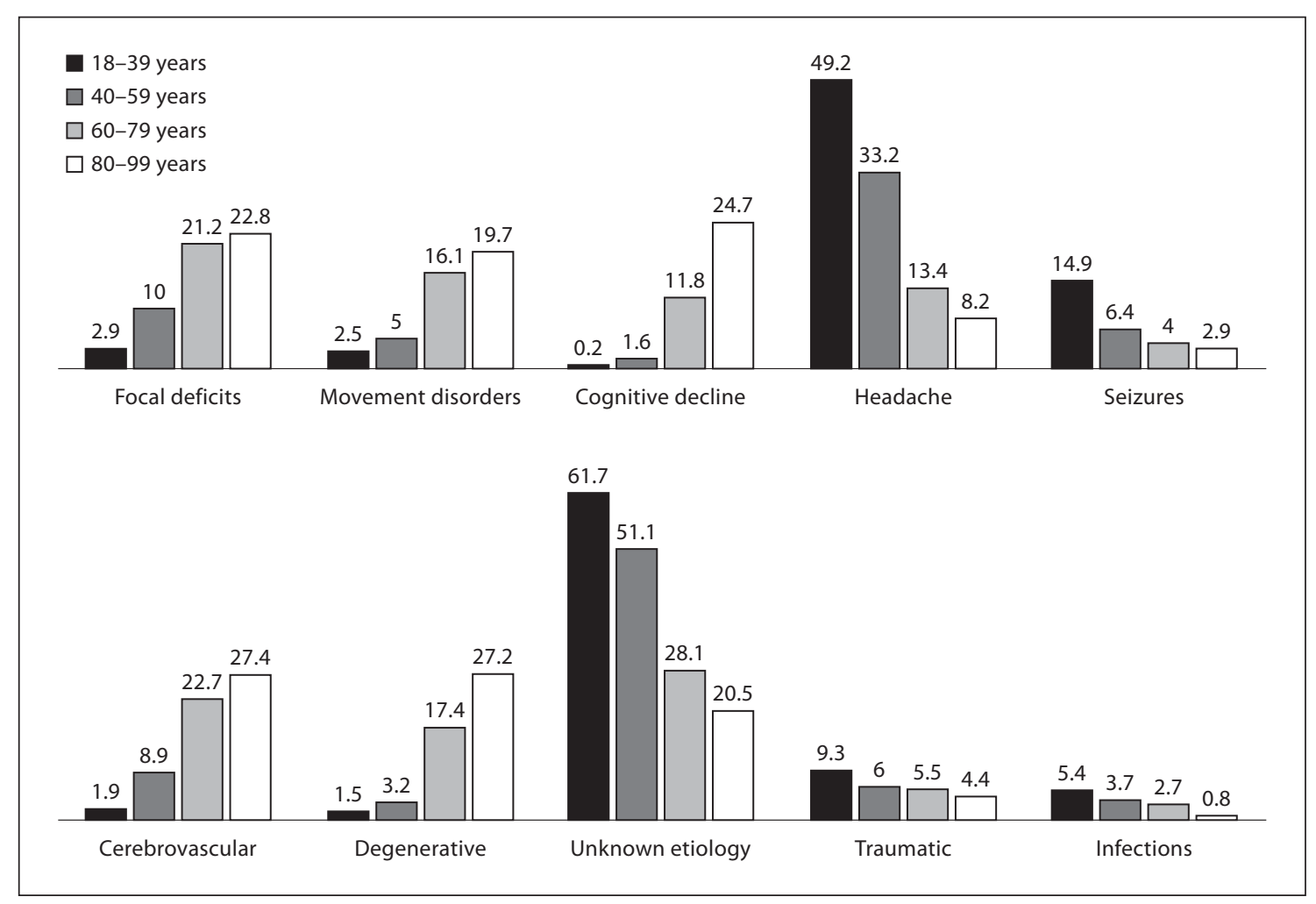

Fig. 1. Relevant differences in reasons for consultation and neurologic diseases according to the age of patients.

\section{Fortuitously Discovered Conditions}

We found silent cerebral infarctions in 120 patients, asymptomatic neurocysticercosis in 104, unruptured vascular malformations in 11, congenital leptomeningeal cysts in 3 , and arrested hydrocephalus in 1 . Silent cerebral infarctions were most often small subcortical infarcts, asymptomatic neurocysticercosis presented as parenchymal brain calcifications in all but 1 patient who had a small cyst in the lateral ventricle, and unruptured vascular malformations were most often saccular aneurysms. 45 of the 120 patients with silent cerebral infarctions consulted for cognitive decline, and 35 for the occurrence of a symptomatic cerebrovascular event (ischemic in 33). Patients with incidentally discovered neurocysticercosis most often consulted for primary headache, although others presented with cerebrovascular or degenerative disorders, head trauma, and brain tumors.

\section{Diseases Appearing in the Follow-Up}

331 patients (mean age $56.3 \pm 18.6$ years; $57 \%$ women) were evaluated for a different condition that the one motivating the initial visit. Most of these new conditions in- cluded: cognitive impairment in 46 , benign positional vertigo in 44 , cerebral infarction in 39 , head trauma in 28 , primary headache in 20 , and protrusion of intervertebral discs in 19. Of the 46 patients developing cognitive impairment in follow-up, 14 had previously been evaluated for a cerebrovascular event, and 10 for Parkinson's disease. Likewise, most of the 39 patients who developed a cerebral infarction on follow-up were initially evaluated by another ischemic stroke or a degenerative disease. Most patients who developed primary headache on follow-up had benign positional vertigo or non-neurologic manifestations during the initial evaluation. Likewise, most patients who developed benign positional vertigo on follow-up initially consulted for primary headache.

\section{Discussion}

We found significant age-related differences in the prevalence of many neurologic disorders. In general, young patients most often consulted for headache/facial pain and seizures, while elderly patients usually present- 


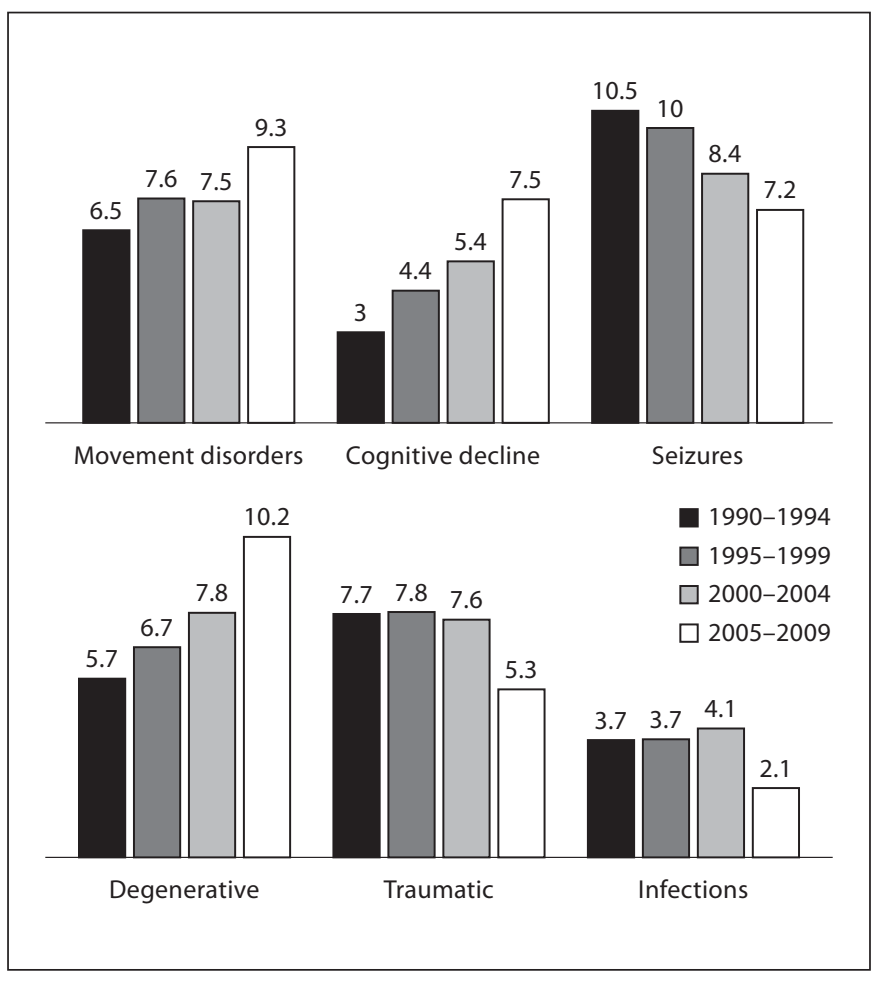

Fig. 2. Relevant differences in reasons for consultation and neurologic diseases according to the year of the first evaluation.

ed with focal neurologic deficits, movement disorders, or cognitive decline. Such differences were directly related to changes in the prevalence of certain neurologic conditions with age. Cerebrovascular and degenerative diseases progressively increased with age, while traumatic diseases, infections of the nervous system, and diseases of uncertain/unknown etiology steadily decreased with advancing age (fig. 1).

We also noted significant differences in the prevalence of some reasons for consultation and neurologic diseases over the 20 years of the study. The most important examples were the reduced number of patients with seizures and the steadily increased number of patients with movement disorders and cognitive decline over the years. The former was directly related to a decreased prevalence of infections of the nervous system (mainly neurocysticercosis-related secondary epilepsy), and the latter to an increased number of patients with degenerative diseases of the nervous system (mainly Parkinson's and Alzheimer's disease) (fig. 2). These findings concur with the concerns of the PAHO (Pan American Health Organization) and other health authorities that have considered that degen- erative diseases and stroke (instead of infections) will be the next health epidemics in Latin America [23, 24]. We have not yet reached the rather high prevalence of dementia among neurologic outpatients reported in some European studies $[9,11]$ but, based on our findings, we may anticipate an increasing number of such patients in the next few years. On the other hand, the prevalence of cerebrovascular disorders in our series did not change over the 20 years of the study despite the assumption that stroke would be now more common than it was 20 years ago. This could be related to the fact that mild stroke cases (as those seen in ambulatory neurologic clinics) may now be assisted by general practitioners and not necessarily come to the neurologist.

We found some regional peculiarities in the prevalence of neurologic disorders. First, the low prevalence of multiple sclerosis in our series $(0.3 \%)$ contrast with that reported from Europe, where this condition has a prevalence higher than $8 \%[5,10]$. This confirms the previously reported very low prevalence of multiple sclerosis in regions near to the equator [25]. Second, we had an important number of patients with neurocysticercosis, a disease that - with only one exception [17] - has not even been mentioned in the existing published series of neurologic outpatients. 190 patients (2.5\%) had symptomatic neurocysticercosis, representing $64.4 \%$ of patients with infections of the nervous system, and $52 \%$ of patients with secondary epilepsy. A decreased prevalence of neurocysticercosis over the years had a significant impact on the prevalence of patients presenting with seizures, as this parasitic disease used to be the most common cause of secondary epilepsy in Guayaquil [26].

The high percentage (47.7\%) of patients with diseases of 'uncertain/unknown etiology' that we found in our series is similar to that reported from other large series of neurologic outpatients $[7,12]$, and is a reflection of the nature of the most common disorders affecting this subset of patients, including primary headaches ( $24 \%$ of our patients), benign positional vertigo, cryptogenic epilepsy, Bell's palsy, vasovagal syncope, and so on (table 5).

Analysis of preexisting and fortuitously discovered conditions, as well as diseases appearing in the follow-up, allowed us to make two main conclusions. First, the main reason for the association between stroke, Parkinson's disease, and mild cognitive impairment seems to be the advanced age of this group of patients (mean age $71.9 \pm$ 9.2 years). Second, the association between primary headache and benign positional vertigo suggests the existence of a particular subset of patients who are prone to develop diseases where symptoms cannot be objectively mea- 
sured. These patients were mostly young women (mean age $36.1 \pm 10.3$ years; $85.7 \%$ women). Whether they could have better been included as non-neurologic (conversive disorders) is a matter of debate; nevertheless, residents and fellows must be aware of this particular group of patients to avoid the practice of unnecessary neurodiagnostic tests.

Comparison of our findings with those reported by others is difficult since most descriptions merge symptoms (i.e. headache) with a group of neurologic diseases (i.e. cerebrovascular) and specific diagnoses (i.e. multiple sclerosis). What is clear from the literature is that headache and seizures are among the main reasons for consultation, that non-neurologic manifestations account for $10-30 \%$ of cases, and that cognitive decline has become a major symptom among neurologic outpatients $[5,11,21]$. Our top reasons for consultation from 1990 to 1994 were headache, seizures and focal neurologic deficits, and we noted that from 2005 on, patients with movement disorders and cognitive decline have outnumbered those presenting with seizures. Such changes were related to both an increasing number of patients with Alzheimer's disease and Parkinson's disease, and a decreasing number of patients with symptomatic neurocysticercosis. Among the different categories of neurologic diseases (excluding diseases of unknown etiology), cerebrovascular and degenerative diseases are top-ranked in most reports $[5,7$, $9,12]$. In our patients, cerebrovascular disorders also ranked first over the years, and we have also noted a significant change in the prevalence of degenerative diseases, which 20 years ago had a low prevalence (5.7\%) and from 2005 on, ranked second only after cerebrovascular disorders (with a prevalence of $10.2 \%$ ).

In summary, our series shows a dynamic pattern of neurologic diseases over the past 20 years. Current distribution of these conditions in our population is more similar to that reported from Europe than it was 20 years ago. While the present study has the potential weakness of having being performed at a single medical center, it has the strength of being performed systematically, using similar diagnostic criteria in all included patients. Moreover, this is the only reported large-scale cohort of neurologic outpatients evaluated over two decades, analyzing not only reasons for consultation and diagnosis, but also the prevalence of preexisting neurologic disorders as well as the appearance of new conditions in the follow-up. Further studies, preferably using a unified classification of symptoms and diseases, are needed to confirm our findings in other populations of the developing world and to compare them with those reported from Europe and the USA.

\section{Disclosure Statement}

The authors have no conflicts of interest to disclose.

\section{References}

1 Naley MA, Elkind MSV: Outpatient training in neurology. History and future challenges. Neurology 2006;66:E1-E6.

-2 D’Esposito M: Profile of a neurology residency. Arch Neurol 1995;52:1123-1126.

-3 Morera-Guitart J: Asistencia neurológica ambulatoria en España. Pasado, presente, ¿futuro? Rev Neurol 2005;41:65-67.

-4 Perkin GD: Pattern of neurological outpatient practice: implications for undergraduate and postgraduate training. J R Soc Med 1986;79:655-657.

5 Hopkins A, Menken M, DeFriese G: A record of patients encounters in neurological practice in the United Kingdom. J Neurol Neurosurg 1989;52:436-438.

-6 Stevens DL: Neurology in Gloucestershire: the clinical workload of an English neurologist. J Neurol Neurosurg Psychiatry 1989;52: 439-446.
7 Perkin GD: An analysis of 7,836 successive new outpatient referrals. J Neurol Neurosurg Psychiatry 1989;52:447-448.

8 MacDonald BK, Cockerell OC, Sander WA, et al: The incidence and lifetime prevalence of neurological disorders in a prospective community-based study in the UK. Brain 2000; 123:665-676.

-9 Huerta-Villanueva M, Baiges-Octavio JJ, Martín-Ozaeta G, et al: Evolución de la demanda de asistencia neurológica ambulatoria y patología atendida en la consulta de neurología de la región sanitaria de Tortosa, Tarragona. Rev Neurol 2005;41:68-74.

10 Maddison P: Neurology training in the United Kingdom: a diagnostic analysis of over 5000 patients. J Neurol 2005;252:605-607.

11 Fragoso M, Aguilar-Barberá M: Análisis de una consulta ambulatoria en el área de Rubí, Barcelona. Rev Neurol 2006:42:58-59.
12 Stone J, Carson A, Duncan R, et al: Who is referred to neurology clinics? The diagnosis made in 3,781 new patients. Clin Neurol Neurosurg 2010;112:747-751.

13 Snijders TJ, de Leeuw FE, Klumpers UM, et al: Prevalence and predictors of unexplained neurological symptoms in an academic neurology outpatient clinic. An observational study. J Neurol 2004;251:66-71.

14 Fink P, Hansen MS, Sondergaard L: Somatoform disorders among first-time referrals to a neurology service. Psychosomatics 2005; 46:540-548.

15 Stone J, Carson A, Duncan R, et al: Symptoms 'unexplained by organic disease' in 1,144 new neurology outpatients: how often does the new diagnosis change at follow-up. Brain 2009; 132:2878-2888.

16 Boongird P, Soranastaporn S, Menken M, et al: The practice of neurology in Thailand. A different type of medical specialist. Arch Neurol 1993;50:311-312. 
-17 Ferri-de-Barros JE, Nitrini R: Que pacientes atende um neurologista? Alicerce de um currículo em neurologia. Arq Neuropsiquiatr 1996;54:637-644.

-18 Siddiqi OK, Atadzhanov M, Birbeck GL, et al: The spectrum of neurological disorders in a Zambian tertiary care hospital. J Neurol Sci 2010;290:1-5.

19 Bergen DC: The world-wide burden of neurologic disease. Neurology 1996;47:21-25.

-20 Singhal BS: Neurology in developing countries. A population perspective. Arch Neurol 1998;55:1019-1021.
21 Ringel SP, Franklin GM, DeLapp HC, et al: A cross-sectional comparative study of outpatient neurologic practice in Colorado. Neurology 1988;38:1308-1314.

22 Narayanaswami P, Hahne JC: Outcome of outpatient neurology consultations: physician's evaluations compared to patients' perceptions. J Neurol Sci 2009;276:175-178.

23 Pan American Health Organization: Health Conditions in the Americas 1994, vol 1. Washington, PAHO Science, 1994, No 549, pp 217-225.
24 Nitrini R, Bottino CMC, Custodio Capuñai NS, et al: Prevalence of dementia in Latin America: a collaborative study of population-based cohorts. Int Psychogeriatr 2009; 21:622-630.

25 Risco J, Maldonado H, Luna L, et al: Latitudinal prevalence gradient of multiple sclerosis in Latin America. Mult Scler 2011;17: 1055-1059.

26 Del Brutto OH, Noboa CA: Late-onset epilepsy in Ecuador: aetiology and clinical features in 225 patients. J Trop Geogr Neurol 1991;1:31-34. 